\title{
Resonance and Anti-Resonance in the Design of Chemotherapeutic Protocols
}

\author{
Z. AGUR \\ Department of Cell Research and Immunology, Life Science Faculty, Tel-Aviv University, Ramat-Aviv, 69978 Tel-Aviv, Israel
}

(Received I October 1996; In final form 28 April 1998)

\begin{abstract}
Analytical and computational results suggest that one can control the growth of cell populations by exposing them to certain dosing frequencies of cell-cycle phase-specific cytotoxic agents. Thus, it has been shown theoretically that a resonance effect, manifesting itself in maximal population sizes, can be created, if the period of the drug pulse and that of the population are commensurable. Based on this theory a method (denoted The Z$M e t h o d$ ) is suggested for improving the efficacy of cancer therapy. The underlying idea of the Z-method is to improve treatment efficacy by selecting treatment periods that create resonance for the limiting normal cell population, and by avoiding resonance for the cancer cells. These theoretical results are supported by in vivo murine experiments, suggesting that intermittent delivery of cell-cycle phase-specific drugs at intervals equivalent to the mean cell-cycle time, might minimize harmful toxicity without compromising therapeutic effects on target cells. A new implementation of the theory, to be denoted the antiresonance effect, is suggested in the present work. In essence, anti-resonance is a practical method of preventing resonance in systems where cancer cell kill needs to be maximized and toxicity to normal cells is marginal. The idea here is to reduce the effective number of cell lines whose period will resonate with the treatment period, by creating a stochastic treatment protocol. An algorithm has been developed for computing the efficacy of specific treatment protocols. The algorithm is independent of the assumptions of the Z-Method. It supports this method by showing theoretically that one can increase treatment success by generating a resonance/anti-resonance relationship between the frequency of drug administration and those of the involved cell populations.
\end{abstract}

Keywords: Cell-cycle phase-specific drug, mathematical model, stochastic process

\section{INTRODUCTION}

Cancer is an increasingly prevalent disease. For example, in Israel, whose total population size is over 4 million, 11,000 new cases were diagnosed in 1989, over 13,000 in 1991 and over 14,000 in 1994. As gene therapy still faces significant hurdles before it becomes an established therapeutic strategy, present control of cancer depends entirely on chemotherapeutic methods.

Chemotherapy is a problem involving many interactive nonlinear processes which operate on different organizational levels of the biological system. It usually involves genomic dynamics, namely, point mutations, gene amplification or other changes on the genomic level, which may result in increasing 
virulence of the neoplasia, or in the emergence of drug resistance. Chemotherapy affects many events on the cellular level, such as cell-cycle arrest at different checkpoints, cell transition in and out of the proliferation cycle, etc. Chemotherapy also interferes with the function of entire organs, most notably, with bone marrow homeostasis. In recent years molecular biology has made an important step forward in documenting many of these processes. Yet, for assessing the contribution of specific molecular elements to the great variety of disease profiles, experimental biology must be provided with tools that allow a formal and systematic analysis of the intricate interaction between the genomic, cellular and cell population processes in the host and in the disease agent. This system is so complex that there is no intuitive way to know how small changes in the drug protocol will affect prognosis. But in spite of this intricacy, attempts to improve chemotherapy have been carried out by "trial and error" alone, with no formal theory underlying the application of specific drug schedules. Such an approach "is apt to result in no improvement, only discouragement and little useful information for future planning" (Skipper, 1986).

The research presented in this article focused on the development of a formal method for increasing the efficacy of cancer treatment. The method was designed by employing a mathematical theory and by testing the theory in laboratory experiments. The experimental results are very encouraging; they suggest that it is feasible to control host toxicity by rational drug scheduling based on the mathematical theory. The mathematical method and the experiments that support it are briefly described below.

\section{THE RESONANCE PHENOMENON IN POPULATION SURVIVAL AND ITS APPLICATION IN CHEMOTHERAPY}

Mathematical models that allow for harsh changes in the environmental conditions suggest that persistence of populations is a non-monotone function of the ratio between the characteristic population periodicity and that of the environmental perturbation. In particular, analysis of nonlinear differential equations models of populations undergoing periodic loss process which is effective only during a portion of the population life-cycle, pinpoints the phenomenon of resonance in population survival. Resonance arises when the period of the imposed loss process coincides with the inherent reproductive periodicity of the population. When this coincidence occurs there is a preferential enhancement of the population growth (Agur, 1982, 1985). This mathematical description has been applied to various population dynamics processes (Agur and Deneubourg, 1985; Agur, 1988), and most notably for devising a method, denoted the Z-Method, for increasing the efficacy of cancer and AIDS treatment (Agur, 1986; Agur, 1989; Agur et al, 1988; Cojocaru and Agur, 1992). The realizations of the phenomenon of resonance in models of cell population growth under chemotherapy is described hereafter.

\subsection{A Simple Stochastic Model for the Effect of Cell-cycle Phase-specific Drugs on the Growth of a Non Age-structured Population}

A simple model for predicting the effect of cellcycle phase-specific drugs on population growth is presented in Agur et al(1988). In this model we assume that cells are sensitive to the drug only during the negligibly short moment of replication:

$$
N(t+\tau)=\alpha N(t)[1-\Delta(t+\tau)]
$$

where $N(t)$ is the number of cells in the drugsensitive phase at time $t$. The duration of the cellcycle is $\tau$, and, since the duration of the susceptible life-phase is taken here as negligibly short, $\tau$ represents also the duration of the resistant phase. The reproduction rate is $\alpha$, and the drug protocol is represented by $\Delta$, which is a stochastic process assuming values 0 and 1 ; the value 1 corresponds to the occurrence of a drug dose that eliminates from the system all the drug-sensitive cells; the value 0 corresponds to ineffective drug doses. 
By applying (1) recursively we obtain

$$
\begin{aligned}
& N(t+n \tau)=\alpha^{n} N(t)[1-\Delta(t+n \tau)] \\
& \quad \times\{1-\Delta[t+(n-1) \tau]\} \ldots[1-\Delta(t+\tau)] .
\end{aligned}
$$

The probability that cells, susceptible to the drug at time $t, 0<t<\tau$, have progeny at time $t+n \tau$ is given by

$$
P\{\Delta(t)=0, \Delta(t+\tau)=0, \ldots \Delta(t+n \tau)=0\}
$$

If $\Delta$ is a Markov process, this equals

$P\{\Delta(t)=0\} \ldots P\{\Delta(t+n \tau)=0 \mid \Delta[t+(n-1) \tau=0]\}$.

If, further we assume that the transitions $\Delta=0 \rightarrow$ $\Delta=1$ and $\Delta=1 \rightarrow \Delta=0$ occur at constant rates, $k$ and $k^{\prime}$, and if the initial equilibrium distribution is

$$
P\{\Delta(0)=0\}=\frac{k^{\prime}}{k+k^{\prime}},
$$

we can obtain the average number of drug-sensitive cells in the $\mathrm{n}^{\text {th }}$ generation in terms of the average duration of the drug pulse and the drug-free interval. Let us denote the average duration, of the states $\Delta=$ 0 and $\Delta=1$ as $\omega=1 / k$ and $\delta=1 / k^{\prime}$, respectively, whose probability densities are given by

$$
\begin{aligned}
& P\{\Delta(t+\tau)=0 \mid \Delta(t)=0\} \\
& \quad=\frac{k^{\prime}}{k+k^{\prime}}+\frac{k^{\prime}}{k+k^{\prime}} e^{-\left(k+k^{\prime}\right) \tau} .
\end{aligned}
$$

In the above model both the states $\Delta=1$ and $\Delta=0$ have a stochastic duration, $\delta$ and $\omega$ respectively. However, as drugs have characteristic halflife it will be more realistic to consider a model in which $\delta$ is a constant, while $\omega$ is an exponentially distributed random variable. The Markov property remains valid for this process provided the time differences, $t_{n}-t_{n-1}, \ldots, t_{2}-t_{1}$ are all larger than the "memory" $\delta$. For $\tau>\delta>\tau / 2$ the transitions, $\Delta=0$ at $t=0 \rightarrow \Delta=0$ at $t=\tau$, can occur only if, either, there is no disturbance in the interval $[0, \tau]$, or, there is one disturbance starting at a point $t$ in the interval $[0, \tau-\delta]$ and no disturbance in the interval $[t+\delta, \tau]$. The probability $P\{\Delta(t+\tau)=0 \mid \Delta(t)=0\}$ is thus the sum of the probabilities for these two possible situations:

$$
\begin{aligned}
P & \Delta(t+\tau)=0 \mid \Delta(t)=0\} \\
& =e^{-\tau / \omega}+\int_{0}^{\tau-\delta} e^{-t / \omega} \cdot e^{-[\tau-(t+\delta)] / \omega} d t .
\end{aligned}
$$

Integrating the sum of these probabilities and implementing in (2) we finally obtain (assuming $N(t)=$ $N_{0}$ for $\left.0 \leq t \leq \tau\right)$ :

$$
\begin{aligned}
& \frac{1}{\tau} \int_{0}^{\tau} N(t+n \tau) d t=\alpha^{n} N_{0} \tau \frac{\omega}{\omega+\delta} \\
& \quad \times\left\{\left[1+\frac{e^{\delta / \omega}}{\omega}(\tau-\delta)\right] e^{-(\tau / \omega)}\right\}^{n-1}
\end{aligned}
$$

By computing (8) we show that the average population size increases with $\delta$ and is maximized for $\tau=\delta+\omega$. The latter result was generalized, and it was analytically shown that for any distribution of the environmental process, the extinction time and the extinction period index in environment $B$ are stochastically lager than those in environment $A$, given that $\left(\tau_{A} / 2\right) \leq \delta<\tau_{B}<\tau_{A}$ (Agur, 1985). Moreover, it can be shown that maximum synchronization of the population with the stochastic environmental process is achieved when $\left(\tau /\left(\delta+\omega_{\min }\right)\right) \rightarrow 1$, where $\omega_{\min }$ is the minimum interval between two successive disturbances.

\subsection{A Probabilistic Model of Asynchronous Age-structured Populations in the Intermittent Presence of Cell-cycle Phase-specific Drugs}

A more detailed model of age-structured cell populations undergoing chemotherapy confirms the above results (Cojocaru \& Agur, 1992). In this model we consider an arbitrary cell of age $a$ at treatment initiation, and define by $p(t, a)$ the probability that the progeny of this cell will survive to moment $t$, so that

$$
p(t+\Delta t, a)=[1-g(t, a) \Delta t] p(t, a) .
$$

In (9) the function $g(t, a)$, the toxic effect of the drug, is a product of the drug's killing efficacy $K(t)$ and the cell susceptibility at time $t, g(t, a)=$ 
$K(t) \chi(t+a)$. The function $\chi(a)$ describes the drug susceptibility of a cell whose age is $a$,

$$
\chi(a)= \begin{cases}1, & a \in \text { susceptible phase } \\ 0, & a \in \text { resistant phase }\end{cases}
$$

If $\xi$ denotes the duration of the drug susceptible cycle phase, and $\rho$ denotes the duration of the drug resistant cycle phase, so that $\tau=\xi+\rho$, and $\tau, \xi$ and $\rho$ are constant, then $\chi(a)$ is periodic, so that $\chi(a+n \tau)=\chi(a), n=1,2, \ldots$

Now look at the state of the cell, or its progeny, at each new dosing; where the dosing interval is $l .(l=\omega+\delta ; \omega$ and $\delta$, defined above, are constants $)$ :

$$
\begin{aligned}
& \{a,(a+l) \bmod \tau,(a+2 l) \bmod \tau, \ldots \\
& (a+(q-1) l) \bmod \tau\} .
\end{aligned}
$$

The average time during $q$ dosings spent in the susceptible phase by the progeny of a cell whose age was $a$ at treatment initiation is given by

$$
A_{q}(s, a)=\frac{1}{q} \cdot \sum_{i=0}^{q-1} \chi((s+a+i l) \bmod \tau) .
$$

$q$ measures the periodicity created by superpositioning the drug protocol on the cell population. If the first dosing hits a cell at age $a$, then the next dosing that will hit its progeny at age $a$ is the $\mathrm{q}^{t h} \cdot q$ is the smallest integer satisfying $(\tau / l)=(q / p)$.

If $\chi(a)$ is periodic and if $l / \tau$ is rational, then $\chi((s+a+i l) \bmod \tau)$ is periodic with period $T=$ $p \tau=q l$ where $p, q$ are co-prime integers. In this case a necessary and sufficient condition for the complete elimination of a cell population is given by (9).

$$
\ln \alpha<\frac{\tau}{l} \int_{0}^{l} K(s) A_{q}(s, a) d s, \quad \text { for all } a,
$$

where $K(s)$ being the efficacy of the drug, namely, the proportion of the cell population eliminated by the drug at any given moment.

Equation (13) clearly demonstrates that the success of the treatment depends not only on the efficacy of the drug and on the availability of susceptible cells but also on the relation between the internal periodicity of the cell population and the dosing interval, that is the periodicity of the environment.
Thus, this model provides a method for predicting the success of specific protocols. Note that the initial age distribution of the cell population does not appear in (13). Thus, we can conclude, that our result holds for cell population with any continuous initial cell age distribution.

Webb (1990) arrives at similar results by the use of models formulated by differential equations. Webb considers an age-structured model and a maturity-structured model of cell population growth. In the maturity-structured model, he shows explicitly that resonances occur exactly at integral and fractional multiples of the common age at which the cells divide. As the division probability density function approaches a delta function, the agestructured model approaches the maturity model. Johnson and Webb (1996) present a rigorous mathematical analysis of the model and demonstrate the resonance phenomenon for a general class of chemotherapy functions. Their analysis shows that the drug dosing interval, rather than the drug dose, is crucial in determining the efficacy of treatment. A different analysis of a similar model (Dibrov et al, 1985) confirms our result by noting that the optimal drug schedule should have a periodicity close to the mean cell-cycle time of the normal cells.

We have analysed the mathematical properties of the Z-Method by applying $\{\phi n\}$ series, where $0<\phi<1$ and $\{\phi n\}$ is the fraction part of $\phi n$. Using continued fractions we have put forward a fast method for computing the treatment duration and the desired number of drug dosings under a ZMethod protocol (Agur and Dvir, 1994). Results on the sequence $\{\phi n\}$ and on continued fraction can be found in Halton (1965), Slater (1967), Swierckowski (1958) and van Ravenstein et al (1990).

\section{IMPLEMENTATION OF THE "RESONANCE PHENOMENON" IN CHEMOTHERAPY - THE Z-METHOD}

The implementation of our mathematical results in clinical chemotherapeutic protocols is not straight forward. One of the main reasons is that drugs 
seldom have a unique target population. Most significantly, many cell-cycle-phase-specific drugs, such as Taxol (paclitaxel), ara-C (Cytosine arabinoside), 5-FU (5-fluorouracyl) etc., administered for eliminating cancer cells, are also toxic to normal human cells. Another important reason is the variability in cell-cycle duration, which is significantly larger in cancer cells (e.g. Retsky et al., 1990; Larsson and Zetterberg, 1995). These two facts, put together with the mathematical results brought forward above, suggest the following policy (the Z-Method):

1. For minimizing damage to normal host tissues the drug dosing frequency should resonate with the limiting host tissue, but should minimize resonance for the cancer cells (Agur et al., 1988, Cojocaru and Agur, 1992). As a first approximation, a policy by which a single fixed dosing frequency is selected, has been investigated numerically and in mice experiments.

2. When the therapeutic agent is not toxic to normal tissues, efficacy of the treatment can be maximized by creating an anti-resonance effect. Now the dosing interval should be taken as a stochastic process. Such a protocol will be most efficacious when the variability in cycle duration of cancer cells is relatively large and when there is low predictability in the natural and the drug imposed transitions from quiescence into the proliferation cycle, and vice versa.

The predictions of the Z-method are supported in part by experiments in mice bearing lymphoma and treated by repeated pulse delivery of the well known S-phase-specific anti-cancer drug, Cytosine arabinoside (ara-C). These experiments show that when the rhythm of drug delivery roughly coincides with the characteristic bone-marrow cell-cycle time, animals survive and myelotoxicity is significantly reduced (Agur et al, 1992). Similar results are obtained in healthy mice treated by the anti-viral cell cycle phase-specific drug AZT (Agur et al, 1991).

To verify if this approach is feasible with methods available in clinical practice, Ubezio et al(1994) determined the optimal intervals between treatments by measuring the effects of single-dose ara- $\mathrm{C}$ on proliferation kinetics of bone marrow cells in mice.
Consequently, the treatment schedule was evaluated by monitoring the kinetic effects of successive doses on toxicity and anti-tumor activity. Results showed that ara-C is toxic to $\mathrm{S}$-phase cells, causing an arrest at the G1/S boundary for about $4-6 \mathrm{~h}$, following which cells cross the S-phase in a nearly synchronized manner. Further results suggest that an optimal window for a second ara- $\mathrm{C}$ dose, designed to preserve the bone marrow proliferating pool, would be at $14-16 \mathrm{~h}$ (for $5 \mathrm{mg}$ ) and $12-14 \mathrm{~h}$ (for $1 \mathrm{mg}$ ) after the first ara-C dosing, since at this time most of the surviving cells will be in the less susceptible phases (G1 and $\mathrm{G} 2 / \mathrm{M}$ ). On the other hand, a time interval of $7-11 \mathrm{~h}$ is expected to impose maximal toxicity to the bone marrow, since at that time surviving cells will be crossing to the susceptible S-phase.

In order to show experimentally that the optimal ara- $\mathrm{C}$ dosing interval in murine chemotherapy is $14 \mathrm{~h}$, it was first proved that the kinetic behavior of bone marrow cells following 2 or 3 ara- $C$ dosings remained similar to that observed after a single dosing, and that cell-cycle kinetics was not affected by the duration of dosing interval. Subsequently, the proportion of proliferating cells that survived different ara-C protocols was evaluated. The latter experiment shows that the second and third ara-C doses, given at $14 \mathrm{~h}$ intervals, did not induce a severe damage to the proliferating bone marrow cells. In contrast, a second dose at the $7 \mathrm{~h}$ and $10 \mathrm{~h}$ interval caused a considerable decrease in the percent of proliferating cells. A close correlation between bone marrow damage and mice survival was observed. $4 / 5$ mice died as a result of treatment with 4 ara-C doses given at $10 \mathrm{~h}$ intervals. Reducing the number of doses to 3 did not decrease mortality. On the other hand, increasing time intervals to $14 \mathrm{~h}$ dramatically improved survival since $5 / 5$ mice survived 3 ara-C doses given at $14 \mathrm{~h}$ intervals, and even 4 doses caused the death of only 1 out of 5 mice.

The next step was to examine the above scheme in tumor bearing mice. In this case, both $4 \times 14 \mathrm{~h}$ and $4 \times 16 \mathrm{~h}$ protocols were tested and compared to a treatment in which 4 drug doses were applied at random (unequal) time intervals. The random protocol included time intervals longer than $16 \mathrm{~h}$ 
and shorter than $14 \mathrm{~h}$ to reach the same overall duration of treatment as in the $14 \mathrm{~h}$ schedule. Here mice were inoculated with 38C-13B lymphoma cells and treatment was initiated 3 days later. Results were indisputable as the $4 \times 14 \mathrm{~h}$ was not only less toxic, but it also delayed tumor development, nearly doubling survival time as compared to the control. The $16 \mathrm{~h}$ protocol was similar to the $14 \mathrm{~h}$ (except for the death of $1 / 4$ mice) defining the width of the bone marrow preserving time window. In contrast, the Control group of untreated mice died within 14 days due to tumor development whereas the random treatment was highly toxic causing $100 \%$ deaths within 7 days.

The above experiments show that protocols employing a dosing interval that is similar to that of the normal bone-marrow cells protect the bone marrow rather well, whereas protocols employing different intervals are extremely toxic. The work suggests that it is feasible to control toxicity in the bone marrow by rational drug scheduling based on the Z-method.

\section{TUNING UP THE Z-METHOD}

An algorithm has been developed for checking the efficacy of specific treatment protocols in well defined host/cancer. The algorithm is completely independent of the Z-Method assumptions. Nevertheless, it supports this method by showing theoretically that one can increase treatment success by generating an intricate resonance/anti-resonance relationship between the frequency of drug administration and those of the involved cell populations. Moreover, using this algorithm one can show numerically that a pulsed drug administration is superior to a continuous administration (bolus) when the treatment optimization problem is defined as minimizing the size of the cancer, while keeping the size of the drug-susceptible host cell population above a well specified threshold (Levy, Hassin, Agur, in preparation).

The algorithm has been implemented for many case studies that vary in the definition of the optimization problem, as well as in the host and in the cancer cell-cycles and pharmacokinetic parameters. Its implementation involves a pre-definition of a parameter set, which includes the distributions of the host and cancer cell-cycles, the distributions of their drug-susceptible phases, the total duration of the treatment and a resolution factor, that determines the length of a single time unit.

The user is asked to set the kill-rate before each treatment. With this rate set, the program simulates the progress of one entire treatment, allowing the user to view the cells status and producing a graphical representation of the cell distribution at the end of the treatment (see, for example, Figure 1). The user then sets the time for the beginning of the next treatment, and the process is repeated.

The main parts of the program are two procedures that simulate the growth of the cells during treatments and when no treatment is given. The life cycles are separated into small time units, and the number of both normal and cancer cells is considered to be constant over that time unit. Here logistic growth rate has been assumed for the normal host cells, while the growth rate of the cancer cells has been taken as exponential. This assumption is reasonable for hematologic malignancies, or for very small solid tumors. However, for larger tumors the law of growth is still a matter of some dispute (e.g., Retsky et al., 1990). Recently, large scale mammography screening trial data have been used to deduce the growth law of primary breast cancer and it was shown that the data are inconsistent with the exponential, logistic and Gompertz laws, but support power law growth (exponent $\approx 0.5$ ) (Hart, Shochat and Agur, 1998). Indeed when chemotherapy of large solid tumors is considered it becomes essential to implement a growth which is slower than exponential.

The graphical representation of the cells status shows the two density functions (representing the numbers of cells with respect to the initial levels) on the same graph. The initial state is that both types of cells have a total amount of one, and all cells are uniformly distributed along the cellcycle. Under treatments and cell growth, the total 


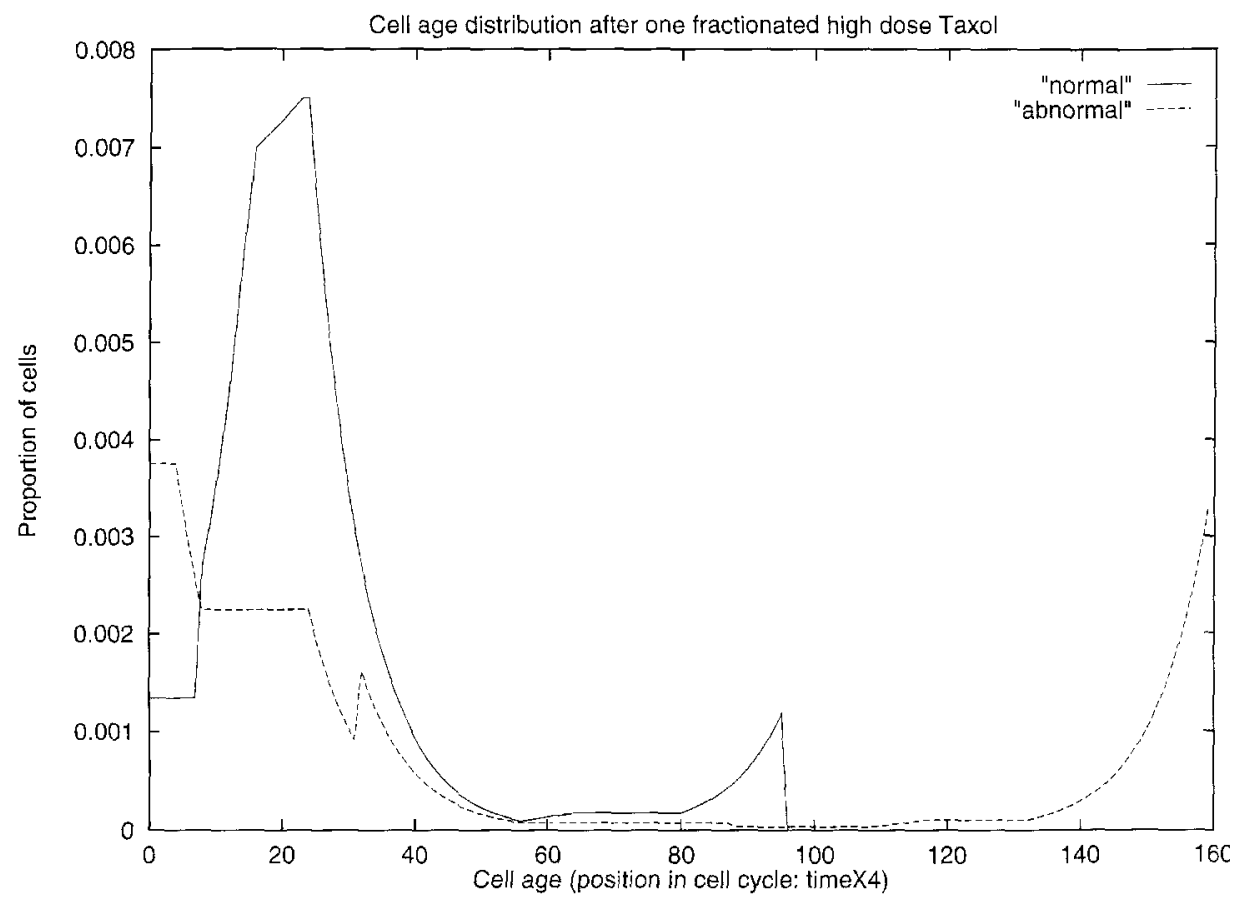

FIGURE 1 Theoretical computations of the age distribution of bone marrow cells and breast cancer cells following a protocol of Taxol (paclitaxel) high dose chemotherapy. The protocol consists of a single dosing, killing $50 \%$ of the cells in the G1 phase or in the $\mathrm{G} 2 / \mathrm{M}$ phase/h, during $10 \mathrm{~h}$. The abscissa represents the age of cells (in the cell cycle) times 4 ; the ordinate represents the proportion of cells. The proportions of host cells and cancer cells surviving the treatment are 0.136848 and 0.147970 , respectively. Assumptions about the duration of the cell-cycle phases: bone marrow cells: $\mathrm{T}(\mathrm{S})-10 \mathrm{~h} ; \mathrm{T}(\mathrm{G} 2 / \mathrm{M})+\mathrm{T}(\mathrm{G} 1)-14 \mathrm{~h}$; breast cancer cells: $\mathrm{T}(\mathrm{S})-13$ h; $\mathrm{T}(\mathrm{G} 2 \mathrm{M})+\mathrm{T}(\mathrm{G} 1)=27 \mathrm{~h}$.

number changes, and the graphs show the change in the number of surviving cells at each moment of the cell-cycles, with respect to the initial uniform distribution of the cells.

The above algorithm has been employed for examining how to improve regimens of high-dose chemotherapy (HDC), currently believed to be one of the most effective strategies for obtaining higher anti-tumor efficacy for breast cancer (BC). In particular, a high-dose $\left(210 \mathrm{mg} / \mathrm{m}^{2}\right) 3 \mathrm{~h}$ infusion of Taxol (half-life of 10 to $12 \mathrm{~h}$ ) is routinely used in adjuvant setting for high-risk patients after mastectomy. Under these regimens dose-limiting myelosuppression is common (e.g., schiller et al., 1994) so that autologous bone marrow transplantation becomes mandatory.

Using the above algorithm the drug's killing effect on cancer cells and on host bone-marrow cells has been calculated for several protocols. Subsequently, a comparison has been made between protocols of a single dosing of Taxol HDC, and protocols of a fractionated dosing with the same or a smaller total dose. In the particular setting depicted in Figure 1 and Figure 2 we assumed that cells in the $\mathrm{G} 1$ and $\mathrm{G} 2 / \mathrm{M}$ phases of the cell-cycle are more susceptible to Taxol than $S$-phase cells (Ubezio, personal communication), and that breast cancer cell cycle lasts $40 \mathrm{~h}$, while that of bone marrow cells is roughly $24 \mathrm{~h}$. The computations, for several sets of pharmacokinetic and cell-cycle parameters, show that by splitting the dose, some advantages on the surviving/killing of two targets which proliferate with different cycles, are possible. In particular, we showed that a HDC protocol in which Taxol exerts high cell kill $(50 \% / \mathrm{h})$ during $10 \mathrm{~h}$ reduces the bone marrow critical cell population to .137 of its initial size and the susceptible cancer population to .148 of its initial size (Figure 1). This drug effect is reversed when the dosing is fractionated so as to resonate with the susceptible bone 


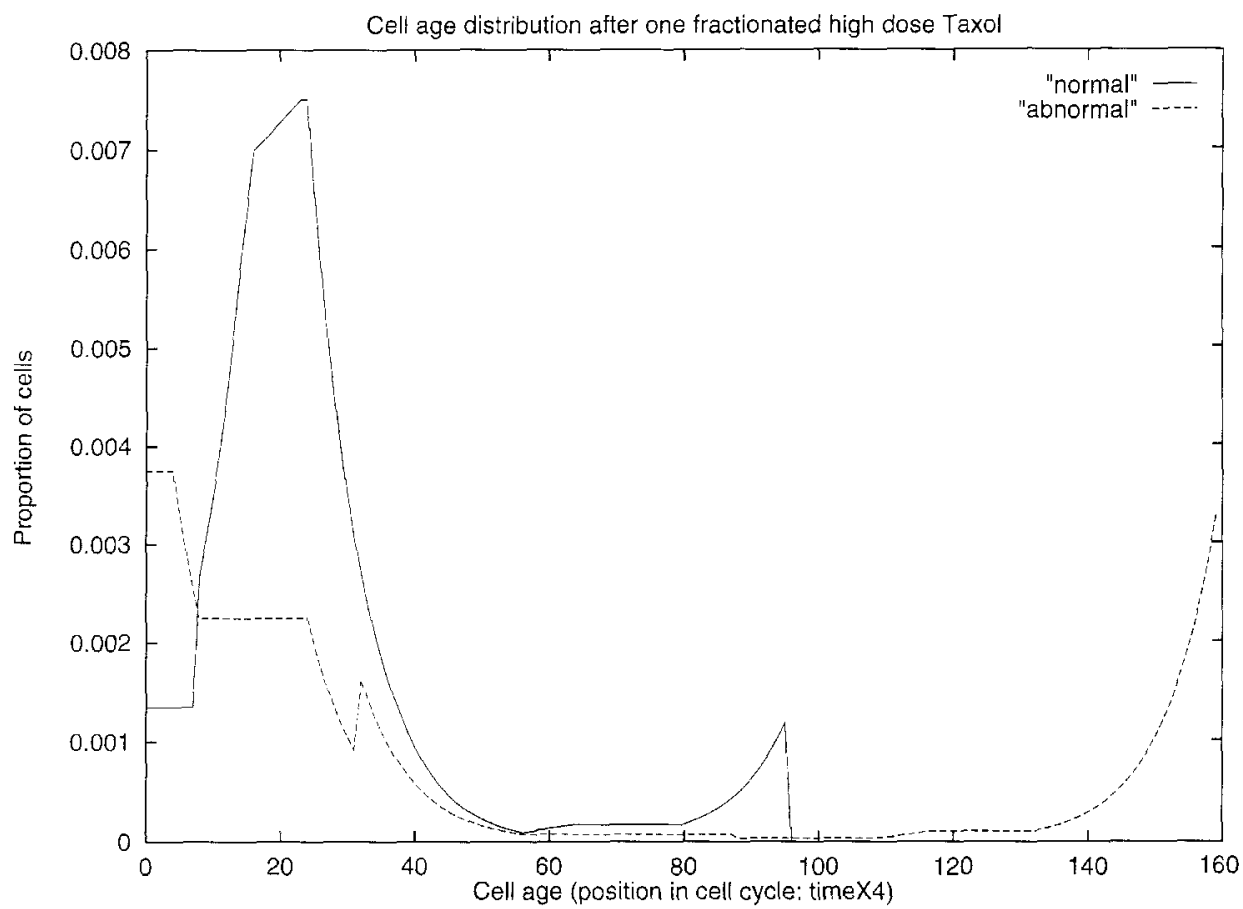

FIGURE 2 Theoretical computations of the age distribution of bone marrow cells and breast cancer cells following a protocol of Taxol (paclitaxel) high dose chemotherapy. The protocol consists of a fractionated dosing, killing $40 \%$ of cells that are in the G1 phase or in the G2/M phase/h (total dose being lower than in Figure 1). First dosing fraction, effective during $8 \mathrm{~h}$, is followed by a $4 \mathrm{~h}$ interval with no cell kill, and a second dosing fraction. killing $40 \%$ of cells/h. which is effective during $2 \mathrm{~h}$. The abscissa represents the age of cells (in the cell cycle) times 4; the ordinate represents the proportion of cells. See legend to Figure 1 for assumptions about the duration of the cell-cycle phases. The proportions of host cells and cancer cells surviving the treatment are 0.177096 and 0.120511 , respectively.

marrow cell-cycle; under these circumstances toxicity to normal cells is reduced while cancer cell kill is augmented (Figure 2).

However, as information on the cell kinetics of human bone marrow is scarce, mainly due to the ethical problems involved in human experiments, the results of our computations need to be considered with caution. A more accurate investigation of the kinetic properties of human bone marrow (with a representation of its main subpopulations, proliferating and quiescent, of stem and progenitor cells), as well as its response to Taxol, will be necessary in order to design and test a precise fractionated protocol of Taxol administration.

\section{Acknowledgments}

The author wishes to acknowledge P. Ubezio, A. Bertuzzi, and A. Gandolfi for helpful discussion, and R. Hassin and S. Levy for computing the effects of Taxol. The work was supported in part by the Israeli Science Ministry through grant No. 9667.

\section{References}

[1] Agur, Z. (1982). Persistence in uncertain environments. Population Biology, Lecture Notes in Biomathematics (eds. H. I. Freedman and C. Strobeck) Springer-Verlag, Heidelberg, pp. 125-132.

[2] Agur. Z. (1985). Randomness, synchrony and population persistence. Journal of Theoretical Biology, 112, 677-693.

[3] Agur, Z. (1986). The effect of drug schedule on responsiveness to chemotherapy. Annals of the New York Academy of Science, 504, 274-277.

[4] Agur, Z. (1988). Population dynamics in harshly varying environments; evolutionary, ecological and medical aspects. Mathematical Ecology (eds. T. G. Hallam, L. J. Gross and S. A. Levin), World Scientific, pp. 440-454

[5] Agur, Z. (1989). A New method for reducing cytotoxicity of the anti-AIDS drug AZT. IMACS Annals on Computing and Applied Mathematics. Biomedical Systems Modeling and simulation, (eds. J. Eisenfeld and D. S. Levine,) J.C. Baltser AG Scientific Publishing Co., pp 59-61. 
[6] Agur, Z. and Deneubourg, J. L. (1985). The effect of environmental disturbances on the dynamics of marine intertidal populations. Theoretical Population Biology, 27, 75-90.

[7] Agur, Z., Arnon, R. and Schechter, B. (1988). Reduction of cytotoxicity to normal tissues by new regimens of phasespecific drugs. Mathematical Biosciences, 92, 1-15.

[8] Agur, Z., Arnon, R., Sandak, B. and Schechter, B. (1991). Zidovudine toxicity to murine bone marrow may be affected by the exact frequency of drug administration. Experimental Hematology, 19, 364-368.

[9] Agur, Z., Arnon, R. and Schechter, B. (1992). Effect of the dosing interval on survival and myelotoxicity in mice treated by Cytosine arabinoside. European Journal of Cancer, 28A(6/7), $1085-109$.

[10] Agur, Z. and Dvir, Y. (1994). Use of knowledge on $\{\phi n\}$ series for predicting optimal chemotherapy treatment. Random and Computational Dynamics, 2(3\&4), 279-286.

[11] Agur, Z., Tagliabue, G., Schechter, B. and Ubezio, P. (1995). AZT effect on the Bone Marrow - a new perspective on the Concorde Trials. Journal of Biological Systems, 3(1), 241-251.

[12] Cojocaru, L. and Agur, Z. (1992). Theoretical analysis of interval drug dosing for cell-cycle-phase-specific drugs. Mathematical Biosciences, 109, 85-97.

[13] Dibrov, B., Zhabotinsky, A., Neyfakh, Y., Orlova, M. and Churikova, L. (1985). Mathematical model of cancer chemotherapy. Periodic schedules of phase-specific cytotoxic-agent administration increasing to selectivity of therapy. Mathematical Biosciences, 73, 1-31

[14] Halton, J. H. (1965). The Distribution of the Sequence $\{n \xi\}$ $(n=1,2,3 \ldots)$, Proceedings of the Cambridge Philosophical Society, 71, 665-670.

[15] Hart, D., Shochat, E. and Agur, Z. (1998). The growth law of primary breast cancer as inferred from mammography screening trials data. British Jour. Cancer, 78(3), Aug. 1998.
[16] Johnson, M. and Webb, G. F. (1996). Resonances in age structured cell population models of periodic chemotherapy, International Journal of Applied Science and Computations, 3(1), 57-67.

[17] Retsky, M. W., et al., (1990). Is Gompertzian or exponential kinetics a valid description of individual human cancer growth? Medical Hypotheses, 33, 95-106.

[18] Schiller, J. H., Storer, B., Tutsch, K., et al. (1994). Phase I trial of 3-hour infusion of paclitaxel with or without granulocyte colony-stimulating factor in patients with advanced cancer. J Clin Oncol., 12(2), 241-248.

[19] Slater, N. B. (1967). Gaps and steps for sequence $n \theta$ mod 1, Proceedings of the Cambridge Philosophical Society, 73, $1115-1122$.

[20] van Ravenstein, T., Winley, G. and Tognetti, K. (1990). Characteristics and the three gap theorem, Fibonacci Quarterly, 28(3), 204-214.

[21] Skipper, H. E. (1986). On mathematical modeling of critical variables in cancer treatment (goals: better understanding of the past and better planning in the furture), Bulletin of Mathematical Biology, 48(3/4), 253-278.

[22] Swierckowski, S. (1958). On Successive Settings of an Arc on the Circumference of a Circle, Fundamental Mathematicae, 1, 127-134.

[23] Ubezio, P., Tagliabue, G., Schechter, B. and Agur, Z. (1994). Increasing 1- $\beta$-D-Arabinofuranosylcytosine efficacy by scheduled dosing intervals based on direct measurement of bone marrow cell kinetics, Cancer Research, 54, 6446-6451.

[24] Webb, G. F. (1990). Resonance phenomena in cell population chemotherapy models, Rocky Mountain Journal of Mathematics, 20(4), 1195-1216. 


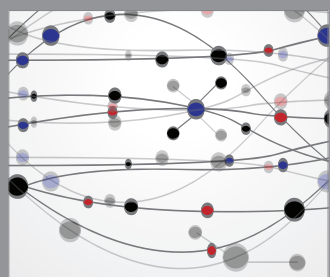

The Scientific World Journal
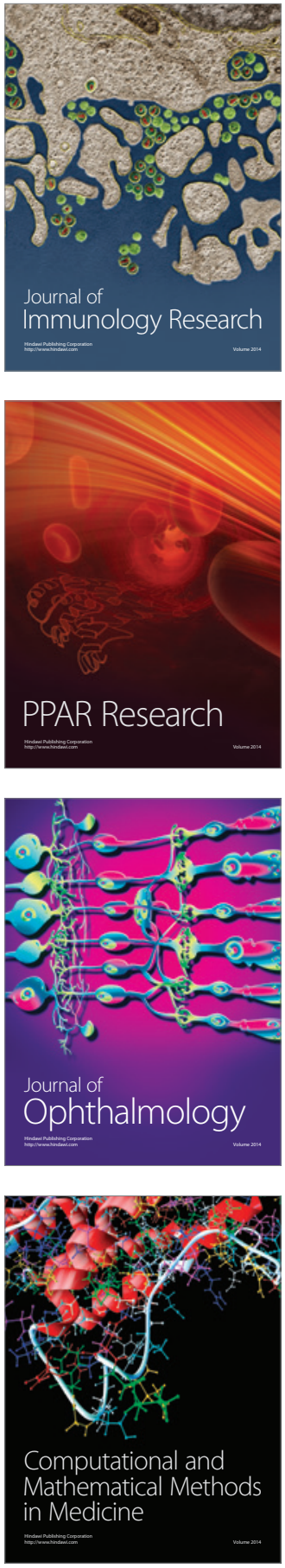

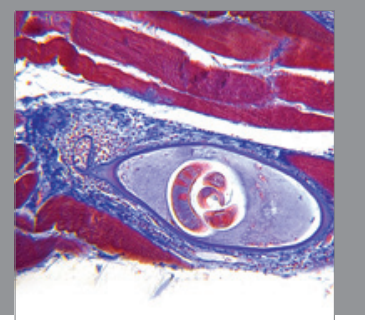

Gastroenterology

Research and Practice
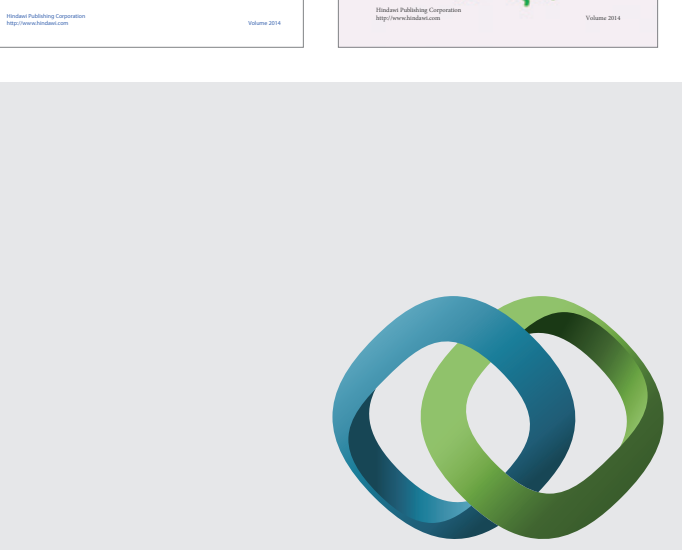

\section{Hindawi}

Submit your manuscripts at

http://www.hindawi.com
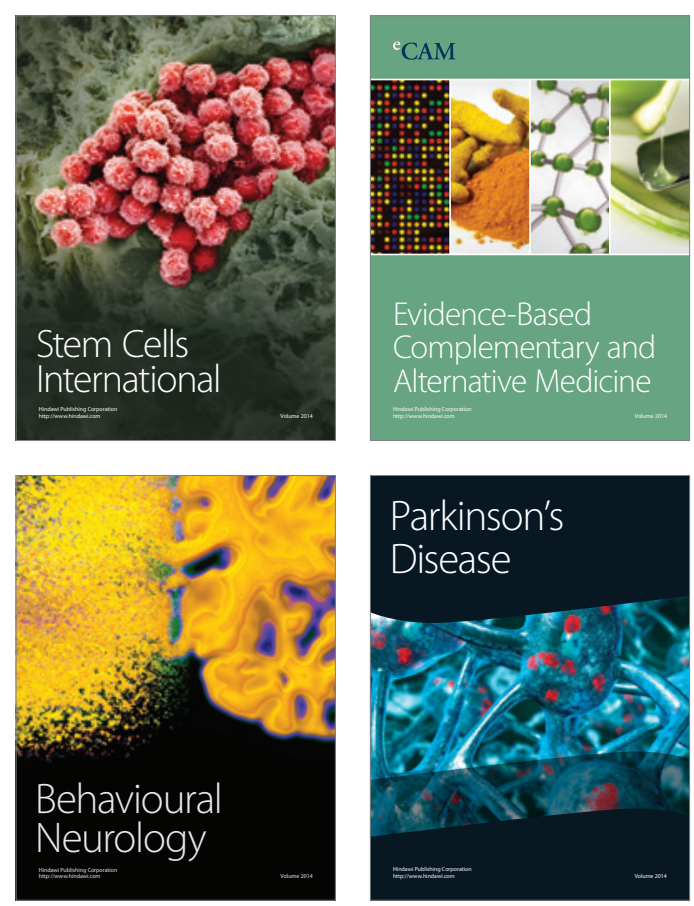

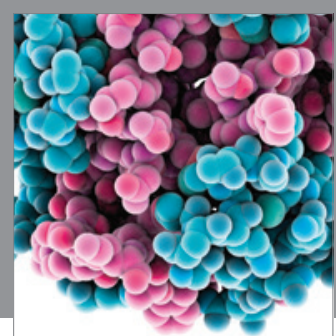

Journal of
Diabetes Research

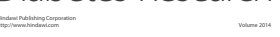

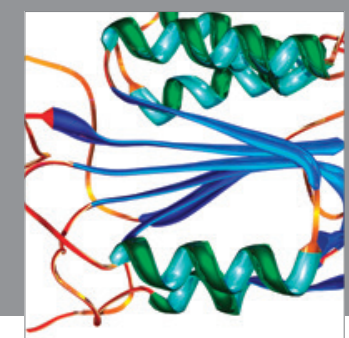

Disease Markers
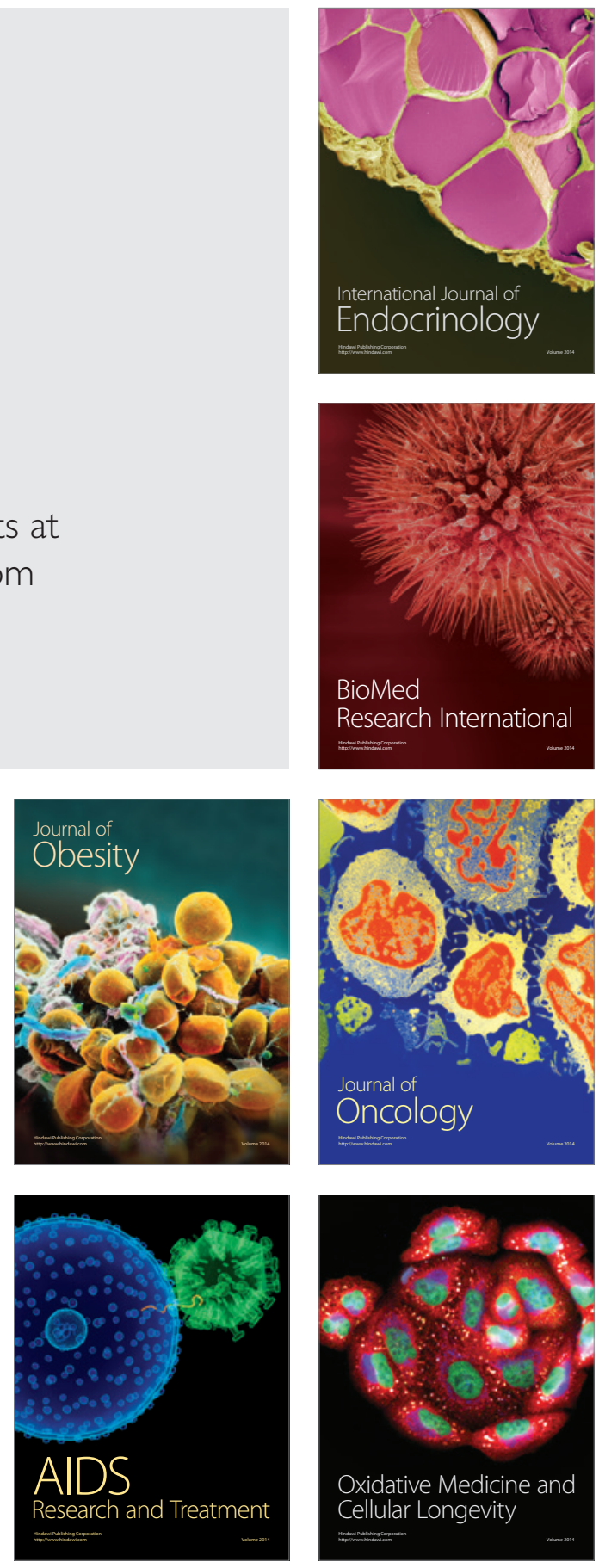